\title{
Experience in the use of clobazam in the treatment of Lennox-Gastaut syndrome
}

\author{
Gabriela Purcarin and Yu-Tze Ng
}

\begin{abstract}
Clobazam is a 1,5-benzodiazepine used successfully worldwide since the 1970s as an anxiolytic and antiepileptic drug. Since its recent Food and Drug Administration (FDA) approval in the United States in 2011 as adjunctive treatment for Lennox-Gastaut syndrome, it has continued to show sustained efficacy and a better safety and tolerability profile compared with other benzodiazepines. The two randomized, controlled studies that led to the US FDA approval, as well as the follow-up multicenter, open-label study of clobazam, showed $\geq 50 \%$ seizure reduction for more than $50 \%$ of Lennox-Gastaut syndrome patients, while none of the other FDA-approved treatments for LGS have demonstrated efficacy rates better than $50 \%$. Clobazam appears to have a safe profile and sustained effectiveness over the first 3 years of use in LGS and other epilepsy syndromes with intractable seizures, which makes it a viable long-term treatment option.
\end{abstract}

Keywords: benzodiazepine, clobazam, drop seizures, Lennox-Gastaut syndrome, open-label trial, pediatric epilepsy

\section{Background}

Lennox-Gastaut syndrome

Lennox-Gastaut syndrome (LGS) is a severe childhood epileptic encephalopathy characterized by:

(1) multiple seizure types, mainly generalized seizures such as tonic, atonic and atypical absence, although partial, myoclonic and generalized tonic-clonic seizures are also observed;

(2) an electroencephalogram (EEG) pattern with diffuse, slow spike-and-wave complexes $(<3 \mathrm{~Hz})$ with characteristic paroxysmal fast rhythms of $10-12 \mathrm{~Hz}$ in sleep;

(3) cognitive dysfunction with psychomotor delay and neuropsychiatric problems [Commission on Classification and Terminology of the International League Against Epilepsy, 1989].

The incidence of LGS is unknown, but is estimated to be between $1 \%$ and $10 \%$ of all childhood epilepsies and it is among the most difficult conditions to treat. It typically develops during early childhood, usually between 3 and 5 years of age, but can be observed anytime between 1 and 8 years of age [Arzimanoglou et al. 2009].

LGS treatment is difficult, challenging, and rarely satisfactory [Lemmon and Kossoff, 2013]. Six antiepileptic drugs (AEDs) are approved by the US Food and Drug Administration (FDA) for LGS at this time: clonazepam, felbamate, lamotrigine, topiramate, rufinamide and clobazam. The majority of LGS cases remain refractory to medical management and require polytherapy and only $10 \%$ of cases are estimated to undergo full seizure remission with available therapies [Crumrine, 2011; Hancock and Cross, 2009]. In addition, comorbidities, including behavioral difficulties and learning disabilities, are common and reported by parents as being among the most troubling symptoms. There are very limited data on the effects of treatment on these variables.

\section{Clobazam}

Clobazam is a 1,5-benzodiazepine and acts through potentiation of gamma-aminobutyric acid type A (GABA-A) receptors in a manner
Ther Adv Neurol Disord 2014, Vol. 7(3) 169-176 DOI: $10.1177 /$ 1756285614521314

(c) The Author(s), 2014. Reprints and permissions: http://www.sagepub.co.uk/ journalsPermissions.nav
Correspondence to: Yu-Tze Ng, MD, FRACP Associate Professor, Department of Neurology University of Oklahoma Health Sciences Center,

711 Stanton L. Young Boulevard, Suite 215 Oklahoma City, OK 73104 USA y-ngRouhsc.edu Gabriela Purcarin, MD Department of Neurology, University of Oklahoma, Oklahoma City, OK, USA 
similar to other benzodiazepines [ $\mathrm{Ng}$ and Collins, 2007; Sankar, 2012]. Its unique chemical design provided by the presence of its nitrogen atoms in the 1 and 5 positions of the diazepine ring instead of the 1 and 4 positions of the traditional benzodiazepines is presumed responsible for the differences in effectiveness and side effect profile [Giarratano et al. 2012]; hence, the origin of its American brand name, 'ONFI', which is derived from a combination of 'one' and 'five'. The efficacy of clobazam as a broad-spectrum anticonvulsant was initially demonstrated in animal studies and it was shown to have reduced sedative and myorelaxant effects relative to the 1,4-benzodiazepines [Steru et al. 1986]. Clobazam is thought to be a selective, partial agonist on GABA-A receptors, with better selectivity toward the GABA-A subunits responsible for anxiolytic and anticonvulsant effects than for those involved in sedation [Rudolph et al. 1999].

It is a long-acting benzodiazepine (median halflife $>36$ hours). Its biologically active metabolite, $\mathrm{N}$-desmethylclobazam, is characterized by similar potency but lesser efficacy and a longer half-life than the parent compound [Walzer et al. 2012; Wheless and Phelps, 2013]. Time to maximum plasma concentration is approximately $1-3$ hours, it is highly protein-bound in serum [Lundbeck, Inc., 2011] and is metabolized in the liver via the cytochrome P450 system. Results of two phase I clinical studies validated the involvement of cytochrome P450 3A4 and 2C19 in clobazam metabolism and no clinically meaningful drugdrug interactions were found, making clobazam a safe choice as adjunctive therapy in patients with LGS, without meaningful changes in dosage necessary in other concomitant AEDs or clobazam itself [Walzer et al. 2012].

Given that LGS is particularly difficult to treat and involves polytherapy, drug-drug interactions between clobazam and other AEDs are a concern. In patients with LGS, clobazam and N-desmethyl clobazam pharmacokinetics were shown not to be affected by the presence of stable dosages of CYP3A4 inducers (phenobarbital, phenytoin, carbamazepine), CYP2C19 inducers (valproic acid, phenobarbital, phenytoin, carbamazepine) or CYP2C19 inhibitors (felbamate, oxcarbazepine) and clobazam is unlikely to affect valproic acid or lamotrigine to a clinically meaningful extent [Walzer et al. 2012]. Although whenever polytherapy is used, there are possible unpredictable and unexpected drug-drug interactions, the following
AEDs did not significantly alter the pharmacokinetics of ONFI or its active metabolite, $\mathrm{N}$-desmethylclobazam, at steady state: valproic acid, phenobarbital, phenytoin, carbamazepine, felbamate, oxcarbazepine [Lundbeck, Inc., 2011].

Clobazam is available as adjunctive therapy for epilepsy and anxiety disorders in many countries. It has been in US clinical development for LGS since 2005. Clobazam (Onfi ${ }^{\mathrm{TM}}$; Lundbeck Inc., IL, USA) was approved in October 2011 by the US FDA for use as adjunctive therapy for the treatment of seizures associated with LGS in patients older than 2 years.

In terms of dosage, clobazam is typically initiated at a low dose, often $5 \mathrm{mg} /$ day or $0.1 \mathrm{mg} / \mathrm{kg} /$ day, and increased at weekly intervals until a minimum effective dose is reached or significant side effects occur. As per the prescribing information, patients $\leq 30 \mathrm{~kg}$ in body weight should be initiated at $5 \mathrm{mg} /$ day and titrated as tolerated to $20 \mathrm{mg}$ /day. Patients $>30 \mathrm{~kg}$ in body weight should be initiated at $10 \mathrm{mg} /$ day and titrated up to $40 \mathrm{mg} /$ day. Dosages $>5 \mathrm{mg} /$ day should be divided into two daily doses. A gentle reminder that FDA indication and 'reallife' therapeutics are not always exactly $100 \%$ compatible, the twice a day dosing recommendation is because the major phase II and III studies were performed with twice-daily dosing. It is very possible that once-daily dosing may be well tolerated and feasible in view of clobazam's long half-life. Clobazam is available in 10 and $20 \mathrm{mg}$ tablets and $2.5 \mathrm{mg} / \mathrm{ml}$ oral suspension. The recent introduction of liquid formulation $2.5 \mathrm{mg} / \mathrm{ml}$ will add to the convenience of administration, initiation and titration of treatment. Doses up to $2 \mathrm{mg} / \mathrm{kg} /$ day (maximum $80 \mathrm{mg} /$ day total dose) divided into twice-daily doses are often required and were shown to be safe [Ng et al. 2012; Lundbeck, Inc., 2011].

No formal guidelines are available regarding converting patients from 1,4-benzodiazepines (i.e. clonazepam) to clobazam. We use a ratio of $1: 10$, consistent with the experience in other practices [Wheless and Phelps, 2013]. At a recent $\mathrm{Onfi}^{\circledR}$ expert speakers' training, an informal survey of around 40 expert neurologists found that a vast majority felt that the $1: 10$ ratio is the most appropriate, with a minority thinking a 1:5 ratio was the most accurate (Y. T. Ng, Personal communication 2013).

As for the safety of clobazam in specific patient populations, there are no adequate studies 
regarding the use of Onfi in pregnant women. Although limited, the available animal data suggest developmental toxicity, including an increased incidence of fetal abnormalities following oral administration of clobazam to pregnant animals at doses similar to those used clinically. Long-term effects on neurobehavioral and immunological function have been reported in rodents following prenatal exposure to benzodiazepines. Therefore, Onfi is a category $\mathrm{C}$ drug and should be used during pregnancy only if the potential benefit justifies the potential risk to the fetus. Onfi is excreted in human milk, but the effects of this exposure on infants are unknown [Lundbeck, Inc., 2011].

\section{Clinical studies}

The safety and efficacy of clobazam as adjunctive therapy for pediatric epilepsy has been well proven over time. It has been used broadly as both a firstline and add-on therapy since its introduction in the 1970 s.

Even prior to approval in the US, numerous studies have supported the safety and efficacy of clobazam as adjunctive therapy for pediatric epilepsy. These studies of clobazam's effectiveness included more than 300 patients with LGS and more than 1300 patients with epilepsy types other than LGS. Clobazam was associated with $>50 \%$ reductions in seizure frequency for $37-61 \%$ of patients, with seizure freedom achieved in $9-14 \%$ of patients [ $\mathrm{Ng}$ and Collins, 2007; Leahy et al. 2011; Silva et al. 2006] and clobazam efficacy lasted for more than 1 year in $42 \%$ of the seizure-free patients [da Silveira et al. 2006].

The efficacy of clobazam was shown to be significant in a large retrospective study by the Canadian Clobazam Cooperative Group [Canadian Clobazam Cooperative Group, 1991]. A total of 877 patients with medically refractory seizures were included, of which, 45 patients had LGS. In total, $43 \%$ of patients with a single seizure type experienced $\geq 50 \%$ reduction in seizure frequency, and from these $13 \%$ became seizure-free. They found that $61 \%$ of patients with multiple seizure types had $\geq 50 \%$ reductions in the frequency of one or more seizure types, and nearly $40 \%$ of patients achieved a $\geq 50 \%$ reduction in the frequency of all their seizure types, including $14 \%$ who experienced seizure-freedom across all seizure types. Seizure types for which clobazam had the greatest efficacy included atonic and myoclonic seizures.

The US FDA's approval of clobazam for LGS was based on two randomized, controlled trials, OV-1002 [Conry et al. 2009] and OV-1012 (also known as the CONTAIN trial) [Ng et al. 2011]. Qualifying patients from the two randomized, controlled studies (OV-1002 and OV-1012) were given the option of continuing clobazam treatment in OV-1004, a multicenter, open-label study of clobazam as adjunctive therapy in patients with LGS.

The study designs for all three trials are provided in Figure 1. The phase II trial (OV-1002) consisted of a 3-week titration and a 4-week maintenance period, followed by either a taper period or extension into an open-label study (OV-1004). The phase III (OV-1012, CONTAIN) trial was designed in a similar fashion and consisted of a 3-week titration period, a 12-week maintenance period, and either a 2- to 3-week taper period or extension into the open-label study (OV-1004).

The OV-1002 study was a phase II trial of 68 patients with LGS between 2 and 30 years of age. Clobazam was found to be well tolerated and shown to decrease the weekly frequency of drop (tonic-atonic seizures) and nondrop seizures. Two clobazam dosages were studied (1.0 and $0.25 \mathrm{mg} / \mathrm{kg} /$ day). The response was dosagedependent, and the $1.0 \mathrm{mg} / \mathrm{kg} /$ day dosage was found to be more efficacious than the $0.25 \mathrm{mg} / \mathrm{kg} /$ day dosage. The drop seizure frequency was reduced by $12 \%$ and $85 \%$ in low-dosage $(0.25$ $\mathrm{mg} / \mathrm{kg} /$ day $)$ and high-dosage (1 $\mathrm{mg} / \mathrm{kg} /$ day $)$ groups, respectively. A total of $83 \%$ of patients in the high-dosage group had $>50 \%$ reduction in weekly drop seizures. The rates of other seizure types also decreased significantly, and global scores showed significant improvements [Conry et al. 2009].

Drop seizures were defined as seizures that involve a change in postural tone which would cause the patient to fall if not supported. Thus, the 'drops' could involve the entire body, trunk or head. They include tonic attacks (drops with body stiffening and falls backward), atonic/akinetic attacks (drops with little or no body stiffening, often bending at the knees with falls sideways or forward) or myoclonic attacks (drops that start with a sudden brief jerk or jerks of face and arms). They are the main focus of anticonvulsant efficacy studies in LGS, 


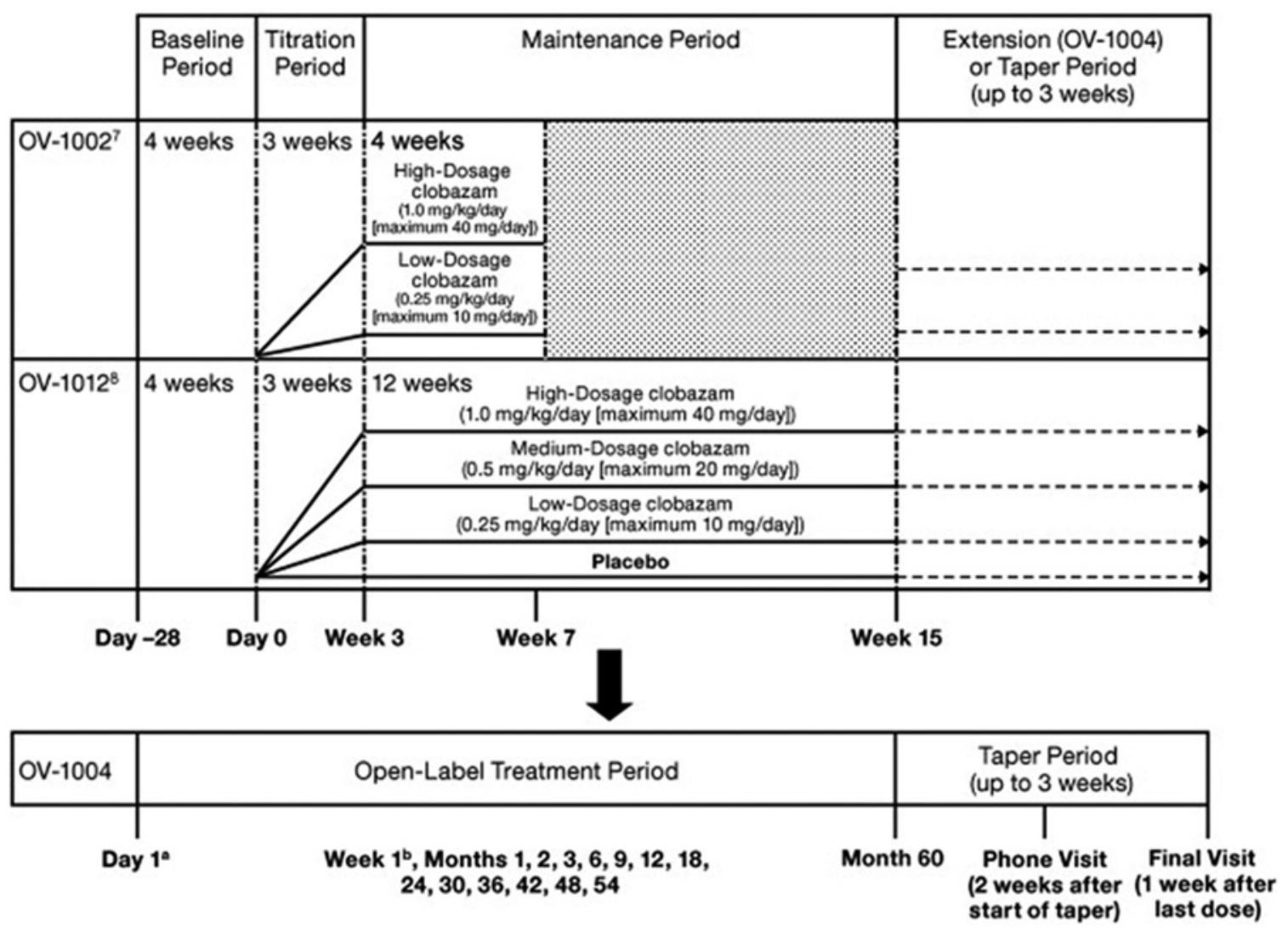

Figure 1. Controlled and open-label study designs.

aEnd of previous blinded Lundbeck-sponsored study and the start of the open-label study.

' 'Week-1 visit' only applied to patients who participated in OV-1012 (patients in OV-1002 had passed the week-1 time point prior to the amendment change that added week-1 procedures).

as they are the most problematic in terms of injury, and they are easily countable (as opposed to other seizure types, i.e. atypical absences).

The phase III trial (OV-1012) was a prospective, double-blind, placebo-controlled study, which established class II evidence about the efficacy and safety of clobazam as adjunctive therapy in LGS [Efficacy and Safety Study of ClObazam in PatieNTs with Lennox-GAstaut SyNdrome (CONTAIN)]. There was a significant dose dependent decrease in seizures, particularly in drop seizures associated with LGS $[\mathrm{Ng}$ et al. 2011]. A total of 238 patients between the age of 2 and 60 years were randomized to placebo or clobazam $0.25,0.5$ or $1.0 \mathrm{mg} / \mathrm{kg} /$ day (maximum total dosages of 10,20 and $40 \mathrm{mg} /$ day). The study consisted of 4-week baseline, 3-week titration and 12-week maintenance phases, followed by a 2- or 3-week taper or continuation to an openlabel extension study. The average weekly drop seizure rates decreased $12.1 \%$ for placebo versus $41.2 \%(p=0.0120), 49.4 \%(p=0.0015)$ and
$68.3 \%(p<0.0001)$ for clobazam $0.25,0.5$ and $1 \mathrm{mg} / \mathrm{kg} /$ day groups, respectively. The responder rates $(\geq 50 \%$ seizure reduction) were $31.6 \%$ (placebo) versus $43.4 \%$ (not statistically significant), $58.6 \%(p<0.05)$ and $77.6 \%(p<0.0001)$ for clobazam $0.25,0.5$ and $1 \mathrm{mg} / \mathrm{kg} /$ day groups. Although there is a higher placebo response than in studies involving other AEDs, the clobazam group still achieved statistical significance with fairly dramatic results.

These results are all the more notable considering that none of the other FDA-approved drugs for the treatment of LGS have demonstrated more than $50 \%$ efficacy rates. Efficacy rates were between $33 \%$ and $50 \%$ for all of the other FDAapproved drugs (admittedly not from open-label studies), while clobazam had $83 \%$ (in the phase II trial) and $77.6 \%$ responder rates (in the phase III trial) noted in the high-dosage clobazam group ( $1 \mathrm{mg} / \mathrm{kg} /$ day) [VanStraten and $\mathrm{Ng}, 2012$ ]. The responder rate implies $\geq 50 \%$ drop seizure reduction. 
All three clobazam dosages decreased weekly rates of total (drop and nondrop) seizures. The decreases were driven mainly by drop seizures, in a dose-dependent fashion. Two patients $(3.5 \%)$ in the placebo group were seizure-free $(100 \%$ decrease from baseline in drop seizure rates), compared with four $(7.5 \%)$, seven $(12.1 \%)$ and $12(24.5 \%)$ patients for the low-, medium-, and high-dosage clobazam groups. The results are notable, as in the high-dosage group, approximately three of four had at least a $50 \%$ decrease and approximately one of four had a $100 \%$ decrease [ $\mathrm{Ng}$ et al. 2011]. Owing to the relatively small numbers of patients in these groups, the study was unable to provide valid statistical comparisons for the $100 \%$ response threshold.

The multicenter open-label extension CLB study continued to show ongoing safety and efficacy. The maximum dosage allowed was $2 \mathrm{mg} / \mathrm{kg} /$ day (maximum $80 \mathrm{mg} /$ day) versus CONTAIN which allowed a maximum dose of $40 \mathrm{mg} /$ day. A total of 267 patients were enrolled and $188(70 \%)$ completed the study. Published interim results of the open-label study at 24 months indicated sustained efficacy for the majority of these patients. The median percentage decrease in weekly drop seizures was $91.6 \%$, with $79.5 \%$ of these patients having a reduction in weekly drop seizures of $\geq 50 \%$ from baseline at 24 months $[\mathrm{Ng}$ et al. 2012]. The percentage of patients with improvement in average weekly seizure rate from previous blinded study baseline increased from month 3 to month 24 for both drop and total seizures. The percentages of patients with a $\geq 50 \%$ drop seizure response rate were $61.5 \%$ at month 3 and $79.5 \%$ at month 24. Clobazam's adverse event profile was consistent with its profile in controlled trials.

In addition, decreases in seizure frequency were accompanied by improved global scores and substantial reductions in use of concomitant AEDs. Both the phase III trial and the long-term openlabel study included physicians' and caregivers' global evaluations of the patients' overall changes in symptoms over time (using a seven-point Likert scale, with $1=$ very much improved and $7=$ very much worse). All three clobazam dosages led to improvements. The percentages of patients who were at least minimally improved ranged from $71.2 \%$ to $81.6 \%$ (physicians' and caregivers' assessments) for clobazam versus $45.5 \%$ to $47.3 \%$ for placebo. Patients who were much improved or very much improved ranged from $41.5 \%$ to $64.9 \%$ (physicians' and caregivers' assessments) for clobazam versus $23.6 \%$ to $25.5 \%$ for placebo [Ng et al. 2011]. In the long-term open-label study, the majority of patients were assessed by the physician as 'very much improved' or 'much improved' at all time points (range: $66.3-82.3 \%$ ), and this percentage increased from month 3 to month 24 . Similarly, the majority of patients were 'very much improved' or 'much improved' at all time points when assessed by the parent/caregiver (range: $61.5-80.5 \%$ ), and this percentage also increased from month 3 to month 24. Although it is a subjective assessment, the global improvement might be related to better seizure control and possible less sedation or decreased use of concomitant AEDs.

Preliminary reports, as yet unpublished, indicate sustained effectiveness with $>50 \%$ decrease in weekly drop seizures at 3 years. The percentage of responders $(>50 \%$ decrease in average drop seizures) was $77.9 \%$ at 3 years, with $38.1 \%$ being free of drop seizures at 3 years. Of note, patients receiving three or more concomitant AEDs at baseline were requiring fewer AEDs at final evaluation (9/36, 25\% with $>3$ AEDs and $27 / 87,31 \%$ with three AEDs). In addition, physicians' and caregivers' assessments indicated an overall improvement of symptoms in $60-80 \%$ of patients [ClinicalTrials.gov identifier: NCT01160770].

Six medications are approved by the US FDA for treatment of LGS: lamotrigine, topiramate, felbamate, rufinamide, clonazepam and clobazam. Figures 2 and 3 represent a summary of placebocontrolled studies with these medications and their efficacy compared with clobazam [VanStraten and $\mathrm{Ng}, 2012$ ].

As it is unlikely that head-to-head clinical trials in this patient population will be conducted, an indirect statistical analysis was made to compare between the efficacies of several adjunctive therapies approved in the United States and Europe for LGS (clobazam, felbamate, rufinamide, topiramate and lamotrigine) [Cramer et al.2013]. High-dosage clobazam $(1.0 \mathrm{mg} / \mathrm{kg} /$ day $)$ was estimated to be more efficacious than other LGS treatments. As efficacy measures were not identical between selected studies, the prespecified primary endpoint of each study was transformed into an effect size via Cohen's $d$ (which is usually interpreted as follows: $d<0.2$, change not detectable; $0.2 \leq d<0.5$, small change; $0.5 \leq d<0.8$, moderate change; and $d \geq 0.8$ : large change). Clobazam was found to have the strongest 


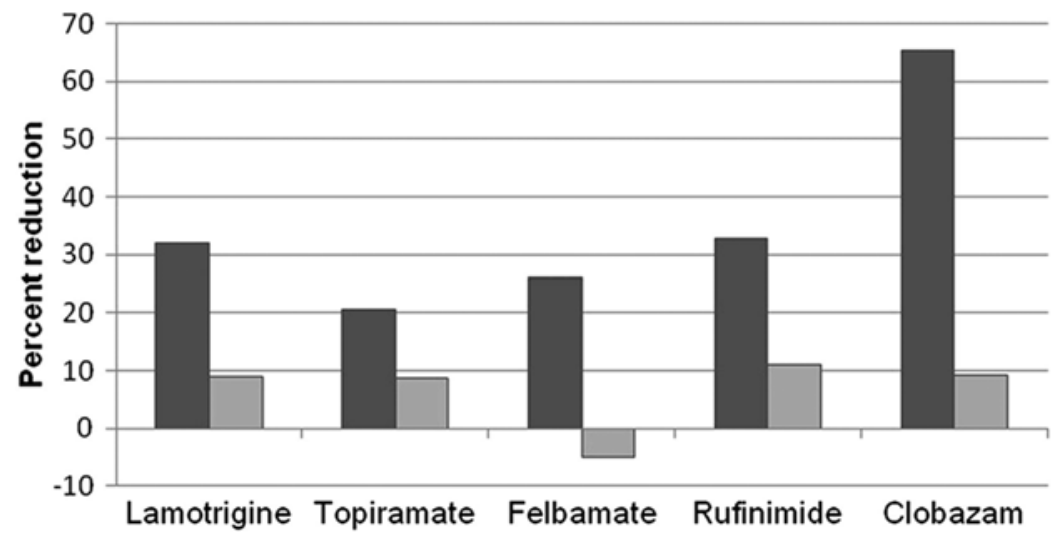

Figure 2. Median reduction in total seizure frequency with antiepileptic drugs approved for Lennox-Gastaut syndrome (dark gray columns) compared with placebo (light gray columns) [The Felbamate Study Group in Lennox-Gastaut Syndrome, 1993; Glauser et al. 2008; Jensen, 1994; Sachdeo et al. 1999; Ng et al. 2011].

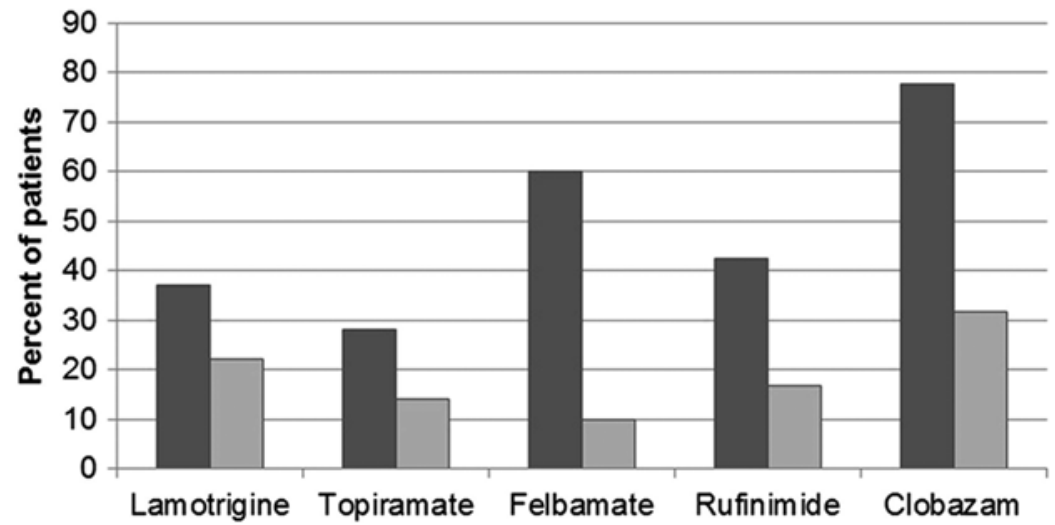

Figure 3. Percentage of patients with more than $50 \%$ reduction in drop seizures with antiepileptic drugs approved for Lennox-Gastaut syndrome (dark gray columns) compared with placebo (light gray columns) [VanStraten and $\mathrm{Ng}, 2012$.

treatment effect versus placebo (effect size 0.80 ), with moderate effects (effect sizes $>0.50$ ) for medium-dosage clobazam $(0.5 \mathrm{mg} / \mathrm{kg} / \mathrm{day})$ and rufinamide. Indirect comparisons of numbers of total seizures and tonic-atonic seizures ('drop attacks') suggested superiority of both clobazam dosages over all comparators. With the other FDA-approved medications for LGS (excluding clonazepam which did not have a phase III efficacy study), the results for total seizure reduction with percentage responders, i.e. $>50 \%$ seizure reduction as compared with placebo were as follows: lamotrigine $33 \%$, placebo $16 \%$; topiramate $33 \%$, placebo $8 \%$; felbamate $31 \%$, placebo $11 \%$ [VanStraten and $\mathrm{Ng}, 2012$ ]. The CONTAIN trial only had results for $>50 \%$ drop seizure reduction responder rates which were: high-dose clobazam $77 \%$, placebo $31.6 \%$ [ $\mathrm{Ng}$ et al. 2011].
Tolerance and safety

In terms of side effects, somnolence, pyrexia and upper respiratory infections were the most frequent adverse events reported for clobazam. Adverse events with $\geq 10 \%$ difference between placebo and any clobazam group in CONTAIN were somnolence, pyrexia, lethargy, drooling and constipation, with somnolence and drooling being dose-related. Discontinuation rates were $6.9 \%$, $12.9 \%$ and $20.3 \%$ of patients in the low- $(0.25$ $\mathrm{mg} / \mathrm{kg} /$ day $)$, medium- $(0.5 \mathrm{mg} / \mathrm{kg} /$ day $)$ and highdosage $(1 \mathrm{mg} / \mathrm{kg} /$ day $)$ groups, respectively. Adverse events leading to discontinuation included lethargy, somnolence, aggression, ataxia, insomnia and fatigue. There were no reports of hepatic failure or death related to clobazam in the phase II and III studies. In the open-label study, 18 patients $(6.7 \%)$ reported adverse events that 
led to premature discontinuation of clobazam. The only adverse events that led to premature discontinuation for more than one patient were pneumonia (three patients) and death of unknown origin or etiology (two patients). There were $10(3.7 \%)$ deaths during the open-label study, nine not related to clobazam and one caused by seizures [Ng et al. 2012; ClinicalTrials.gov identifier: NCT01160770].

\section{Discussion}

The intractable, variable and progressive nature of LGS makes it one of the most challenging seizure disorders to diagnose and treat. The efficacy and safety of clobazam is well established in the treatment of pediatric epilepsies and LGS in particular. Clobazam was approved by the US FDA in October 2011 for use as adjunctive therapy for the treatment of LGS in patients 2 years of age and older based on two randomized, controlled trials and has been shown to have sustained efficacy for patients treated for $\geq 3$ years in an openlabel trial. As a 1,5-benzodiazepine, clobazam has a better safety and tolerability profile and a lesser potential for drug-drug interactions relative to other benzodiazepines. Its efficacy was well proven in the two randomized, controlled US studies with a dosage-related effect on reducing all seizure types, especially drop seizures (tonicatonic). The results are all the more notable considering that none of the other FDA-approved drugs for the treatment of LGS have demonstrated more than $50 \%$ efficacy rates, while $83 \%$ and $77.6 \%$ responder rates $(\geq 50 \%$ drop seizure reduction) were shown in the high-dosage clobazam group $(1 \mathrm{mg} / \mathrm{kg} /$ day $)$. In addition, overall patient quality of life was subjectively improved with clobazam treatment, from global evaluations by both the physician and caregiver/patient.

No head-to-head comparative clinical trials between different AEDs are available, but indirect comparisons demonstrated superiority of clobazam over other AEDs in controlling drop seizures. However, the treatment needs to be individualized and it remains unknown exactly which medication is most effective, and in which patients [Cramer et al. 2013].

Clobazam appears to have a safe profile and sustained effectiveness over at least the first 3 years of use in LGS and other epilepsy syndrome with intractable seizures, which makes it a viable longterm treatment option. Given the safety profile and efficacy, clobazam should be at least considered prior to any invasive procedure or treatment [SeifEddeine and $\mathrm{Ng}$, 2012]. Based on available therapies and accounting for risks and benefits, clobazam may be initiated after one or more appropriate AEDs have been sufficiently tried, particularly to treat drop seizures in LGS, but also considered for treatment of other seizure types in all epilepsies. In our opinion clobazam is probably the most effective medication for drop seizures and although effective for all seizure types, it is probably less so amongst the nondrop seizures. Given its safety profile it may be and has been used first line in some countries, e.g. India. However it is unlikely that in the US it will be used first line in the near future given its FDA indication (even if not all of us follow FDA indications all of the time).

\section{Conflict of interest statement}

Dr Purcarin has nothing to disclose. Dr $\mathrm{Ng}$ has served as a consultant, study investigator, and scientific advisory board member for Lundbeck, Inc. and has received consulting fees, travel funding, and speaker honoraria. This study did not receive any funding or manuscript support from any company.

\section{Funding}

This research received no specific grant from any funding agency in the public, commercial, or notfor-profit sectors.

\section{References}

Arzimanoglou, A., French, J., Blume, W., Cross, J., Ernst, J., Feucht, M. et al. (2009) Lennox-Gastaut syndrome: a consensus approach on diagnosis, assessment, management, and trial methodology. Lancet Neurol 8: 82-93.

Canadian Clobazam Cooperative Group (1991) Clobazam in treatment of refractory epilepsy: the Canadian experience. A retrospective study. Epilepsia 32: 407-416.

Commission on Classification and Terminology of the International League Against Epilepsy (1989) Proposal for revised classification of epilepsies and epileptic syndromes. Epilepsia 30: 389-399.

Conry, J., Ng, Y., Paolicchi, J., Kernitsky, L., Mitchell, W., Ritter, F. et al. (2009) Clobazam in the treatment of Lennox-Gastaut syndrome. Epilepsia 50: 1158-1166.

Cramer, J., Sapin, C. and François, C. (2013)

Indirect comparison of clobazam and other therapies 
for Lennox-Gastaut syndrome. Acta Neurol Scand 128: 91-99.

Crumrine, P. (2011) Management of seizures in Lennox-Gastaut syndrome. Paediatr Drugs 13: $107-118$.

da Silveira, M., Montenegro, M., Franzon, R., Guerreiro, C. and Guerreiro, M. (2006) Effectiveness of clobazam as add-on therapy in children with refractory focal epilepsy. Arq Neuropsiquiatr 64: 705-710.

Glauser, T., Kluger, G., Sachdeo, R., Krauss, G., Perdomo, C. and Arroyo, S. (2008) Rufinamide for generalized seizures associated with Lennox-Gastaut syndrome. Neurology 70: 1950-1958.

Giarratano, M., Standley, K. and Benbadis, S. (2012) Clobazam for treatment of epilepsy. Expert Opin Pharmacother 13: 227-233.

Hancock, E. and Cross, H. (2009) Treatment of Lennox-Gastaut syndrome. Cochrane Database Syst Rev 8: CD003277.

Jensen, P. (1994) Felbamate in the treatment of Lennox-Gastaut syndrome. Epilepsia 35(Suppl. 5): 54-57.

Leahy, J., Chu-Shore, C. and Fisher, J. (2011) Clobazam as an adjunctive therapy in treating seizures associated with Lennox-Gastaut syndrome. Neuropsychiatr Dis Treat 7: 673-681.

Lemmon, M. and Kossoff, E. (2013) New treatment options for Lennox-Gastaut syndrome. Curr Treat Options Neurol 15: 519-528.

Lundbeck, Inc. (2011) ONFI (clobazam) [prescribing information]. Deerfield, IL: Lundbeck, Inc. Available at: http://www.lundbeck.com/upload/us/files/pdf/ Products/Onfi_PI_US_EN.pdf (accessed 14 January 2014).

$\mathrm{Ng}$, Y. and Collins, S. (2007) Clobazam. Neurotherapeutics 4: 138-144.

Ng, Y., Conry, J., Drummond, R., Stolle, J. and Weinberg, M. for the OV-1012 Study Investigators (2011) Randomized, phase III study results of clobazam in Lennox-Gastaut syndrome. Neurology 77: 1473-1481.
Ng, Y., Conry, J., Paolicchi, J., Kernitsky, L., Mitchell, W., Drummond, R. et al. (2012) Long-term safety and efficacy of clobazam for Lennox-Gastaut syndrome: 2-year results of an open-label extension study. Epilepsy Behav 25: 687-694.

Rudolph, U., Crestani, F., Benke, D., Brünig, I., Benson, J., Fritschy, J. et al. (1999) Benzodiazepine actions mediated by specific gamma-aminobutyric acid(A) receptor subtypes. Nature 401: 796-800.

Sachdeo, R., Glauser, T., Ritter, F., Reife, R., Lim, P. and Pledger, G. (1999) A double-blind, randomized trial of topiramate in Lennox-Gastaut syndrome: Topiramate YL Study Group. Neurology 52: 1882-1887.

Sankar, R. (2012) GABAA receptor physiology and its relationship to the mechanism of action of the 1,5-benzodiazepine clobazam. CNS Drugs 26: 229-244.

Seif-Eddeine, H. and Ng, Y. (2012) Clobazam for patients with Lennox-Gastaut syndrome and epilepsy. Expert Rev Neurother 12: 385-393.

Silva, R., Montenegro, M., Geurreiro, C. and Geurreiro, M. (2006) Clobazam as add-on therapy in children with epileptic encephalopathy. Can $\mathcal{F}$ Neurol Sci 33: 209-213.

Steru, L., Chermat, R., Millet, B., Mico, J. and Simon, P. (1986) Comparative study in mice of ten 1,4-benzodiazepines and of clobazam: anticonvulsant, anxiolytic, sedative, and myorelaxant effects. Epilepsia 27(Suppl. 1): S14-S17.

The Felbamate Study Group in Lennox-Gastaut Syndrome (1993) Efficacy of felbamate in childhood epileptic encephalopathy (Lennox-Gastaut syndrome). N Engl f Med 328: 29-33.

VanStraten, A. and Ng, Y. (2012) Update on the management of Lennox-Gastaut syndrome. Pediatr Neurol 47: 153-161.

Walzer, M., Bekersky, I., Blum, R. and Tolbert, D. (2012) Pharmacokinetic drug interactions between clobazam and drugs metabolized by cytochrome P450 isoenzymes. Pharmacotherapy 32: 340-353.

Wheless, J. and Phelps, S. (2013) Clobazam: a newly approved but well-established drug for the treatment of intractable epilepsy syndromes. $\mathcal{F}$ Child Neurol 28: 219-229. 